FORUM FORUM
FORUM
FORUM is intended for new ideas or new ways of interpreting existing information. It provides a chance for suggesting hypotheses and for challenging current thinking on ecological issues. A lighter prose, designed to attract readers, will be permitted. Formal research reports, albeit short, will not be accepted, and all contributions should be concise with a relatively short list of references. A summary is not required.

\title{
Mechanisms promoting plant coexistence: can all the proposed processes be reconciled?
}

\author{
Sébastien Barot, LEST - IRD, 32 Avenue H. Varagnat, FR-93143 Bondy Cedex, France \\ (sebastien.barot@bondy.ird.fr). - Jacques Gignoux, UMR 7625, Fonctionnement et Evolution des Systèmes \\ Ecologiques, Ecole Normale Supérieure, 46 Rue d'Ulm, FR-75230 Paris cedex 05, France.
}

The coexistence of many species in plant communities is difficult to explain because plants use a priori the same resources. After several decades of theoretical and empirical work many mechanisms have been proved to foster plant coexistence. Using the bibliography we classify these mechanisms according to three criteria. (1) Mechanisms of coexistence are equalising, i.e. they diminish the fitness differences between species, or stabilising, i.e. they insure that a species driven to a very low density can again invade a plant community. Only stabilising mechanisms promote stable coexistence. (2) Stabilising mechanisms are always based on the partitioning of a resource, which ultimately reduce to space or time. (3) Both exogenous and endogenous processes are sources of heterogeneity and variability which enable coexistence through space and time partitioning. Processes involved in endogenous heterogeneity enable coexistence in many ways because niches and differentiation of niches between species are selfgenerated at the same time by plant communities. Although most mechanisms of plant coexistence have probably been discovered, four questions should be still addressed: Is there a link between mechanisms of coexistence and ecosystem functioning and community stability? Which mechanisms dominate real communities? How stable is coexistence in real communities? How do mechanisms of coexistence and evolution interact?

One of the first 'laws' discovered in ecology, the principle of competitive exclusion, states that species having the same ecological niche cannot coexist (Hutchinson 1959). They compete for the same resources, and cannot escape this competition. Thus, the species that is the best competitor, i.e. the one which can persist at the lowest level of resource availability, is predicted to exclude all the other (Tilman 1982). To explain the coexistence of numerous animal species it is then easy to invoke their use of different resources: each resource, for example a prey type for a carnivore, can be used as a new axis to define the ecological niche.

The coexistence of numerous species in plant communities is a priori much more mysterious since all photosynthetic plants compete for the same few resources, i.e. water, light, $\mathrm{CO}_{2}$ and soil nutrients. This issue is particularly tricky for phytoplankton (Hutchinson 1961) and for tree communities in tropical forests (Janzen 1970, Connell 1971). In both cases a high number of species with seemingly identical niches and life form coexist. Since this issue has been raised numerous mechanisms of plant coexistence have been proposed and the relevance of these mechanisms has been demonstrated, originally using theoretical models. Although these mechanisms are numerous (Fig. 1), we show that they can be classified using a few fundamental principles (Fig. 2). This should in turn help understanding better plant coexistence and clarifying which avenues should be developed to go further.

\section{Space and time are the ultimate resources}

To persist in a community, a species must find moments when, and places where it can send seeds that will be able to germinate and give birth to new individuals which in turn will find enough resources and space to survive and develop into new adults. This statement is tautological but leads to the conclusion that both space and time can be considered as resources (Tilman and Pacala 1993, Chesson 2000b) and that these two resources recapitulate all other resources. As for trophic resources, when a plant species uses a given fraction of the surface available, especially when this species is the best competitor, this space cannot be used by other species. Similarly, when a plant species occupies sites during a 
given fraction of time these sites are not available to other species during this period (unless the species is competitively inferior and can be displaced). As for trophic resources, the resources represented by space and time are renewed. Here the renewal of these resources takes place when individuals of the considered species die and leave some available space at a given time. Consequently, space and time as resources meet Abram's general definition of resources (Abrams 1988) and their renewal is directly linked to population dynamics. Of course, time and space should not be considered separately, the true resource is constituted by the combination of space and time.

It is now widely recognised that many species can coexist partitioning space according to the heterogeneity of some traits of the physical environment (Chesson 2000a). Each species is the best competitor on a fraction of the available space (in other words, in some of local patches), which diminishes interspecific competition relatively to intraspecific competition and enables coexistence. It is similarly recognised that plant species can coexist partitioning time according to some variable traits of the environment (Chesson and Warner 1981). If each species is the best competitor during a portion of the time coexistence is again possible. Taken together, plant can coexist just by the exploitation of different portions of time and space, which can be considered as spatio-temporal recruitment windows. So far, we have not explained the origin of spatial heterogeneity and temporal variability in the physical environment.

First, purely physical processes make an ecosystem spatially heterogeneous (for example, pedogenesis or topography creates some soil heterogeneity), and temporally variable (climatic variability). This can be called coexistence through exogenous heterogeneity or variability (Fig. 1A). Second, plant dynamics, on its own, generates environmental heterogeneity since plants are ecosystem engineers (Jones et al. 1997). Plants compete with each other, absorbing light, soil nutrients and water. What is absorbed is eventually no longer available for other individuals, but constitutes first a modification of the physical environment. Individuals of each plant species are likely to modify their environment locally, in their own way. Consequently, plant dynamics is likely to generate enough environmental heterogeneity to explain the coexistence of many species. This can be called coexistence through endogenous heterogeneity (Pacala and Levin 1997, Fig. 1A). The mechanisms of this type of coexistence are detailed in next section. The dynamics of plant communities can also generate some temporal variability. As soon as population densities are not constant their retroaction on the physical environment should foster some environmental variability. Although this idea of endogenous variability as a factor of plant coexistence (Fig. 1) has not been developed as much as the idea of endogenous heterogeneity it has been shown that plant populations can cycle without cycles being forced by an external constraint (Bauer et al. 2002) or that cycles could be generated by interactions with herbivores as soon as time-lags and overcompensation mechanisms are present (Crawley 1996) as shown by classical predator-prey models.

In this context, coexistence based on plant species having different regeneration niches (Grubb 1977), is just a way to emphasise that whole life cycles have to be taken into account, and that seedlings are likely to have different ecological requirements from adults ones. This mechanism of coexistence is again based on time or space partitioning involving endogenous or exogenous temporal variability or spatial heterogeneity (Fig. 1A). Similarly, the idea of regional coexistence is based on space partitioning, this time at a larger scale (Mouquet and Loreau 2002): if the different local communities of a region occupy different environments, and if each species of the regional pool is the best competitor in one of these environments, $\mathrm{n}$ species can coexist in a metacommunties composed of $\mathrm{n}$ communities. Local diversity is then influenced by dispersal between communities.

\section{Self-generated spatial heterogeneity as a source of many coexistence mechanisms}

Terrestrial plants are sessile organisms which modify their environment locally according, for example, to the size of their canopy or their root system. It has been shown that local depletion of soil through limited resource transport promotes coexistence (Huston and DeAngelis 1994, Fig. 1A). Besides, local interactions between plant individuals, due to local environmental modifications, lead to complex plant spatial structures. These structures are particularly difficult to understand and predict because of the existence of a feedback loop (Bolker and Pacala 1997, Law and Dieckmann 2000): (1) plant spatial patterns determine the numbers of plant neighbours of the different plant species; (2) neighbours locally modify the access of plants to physical resources on an individual basis; (3) this influences fecundity, growth and survival of individuals differentially, according to the characteristics of their neighbourhood; (4) this process determines finally the dynamics and the spatial pattern of the different plant populations. It must be pointed out that spatial patterns determine also the distances between conspecific and heterospecific neighbours and thus influence directly the intensity of intraand inter-specific competition. More generally, to enable coexistence through a mechanism based on endogenous heterogeneity, a process has to create 'holes' (Murell and Law 2003) in the population of the best competitor to enable the recruitment of new individuals of the poorer competitor. We call these processes coexistence through deterministic recruitment limitation (doted-line box, 


\section{Mechanisms of plant coexistence}

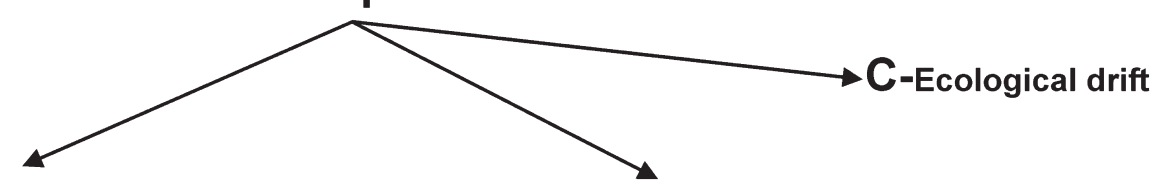

A-Stabilizing processes:

Stable coexistence

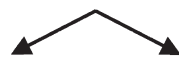

Space as a resource

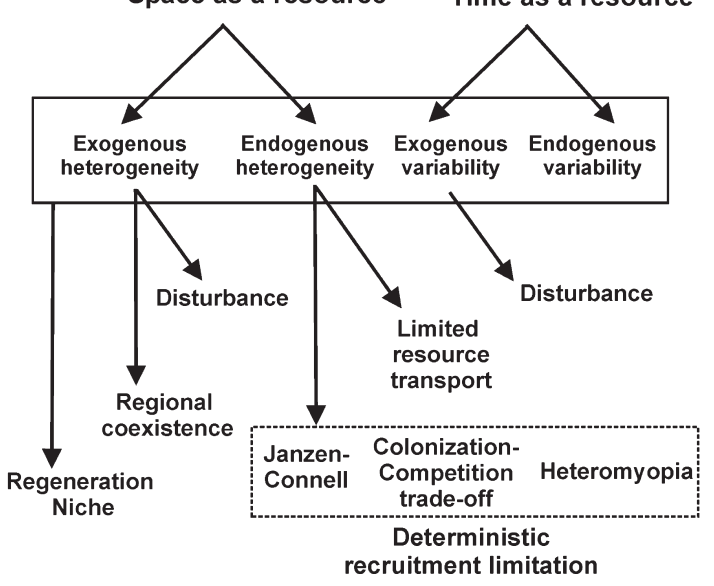

B-Equalizing processes: Unstable coexistence

Processes that slow the deterministic exclusion dynamics

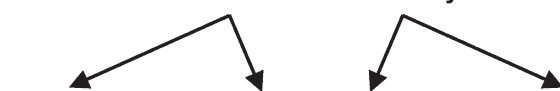

Small fitness Intraspecific Demographic differences, aggregation stochasticity trade-off

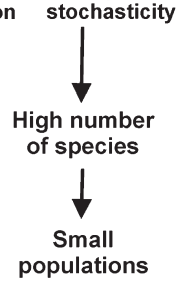

Complexe endogenous

Complexe endogen
spatial structure

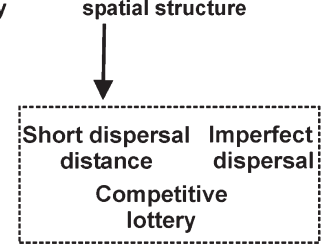

Recruitment limitation as a rule: spatially-mediated demographic stochasticity

Fig. 1. Classification of mechanisms of plant coexistence. (A) Stabilising mechanisms which lead to stable coexistence. (B) Equalising mechanisms which lead to unstable coexistence and only slow down competitive exclusion. (C) Ecological drift. The solid line box encompasses all the fundamental processes that are involved in the regeneration niche hypothesis. The doted line boxes correspond to processes that result in complicated spatial structures in plant populations and promote recruitment limitation. Some of these processes are stabilising (A), other are equalising (B). Traditionally stable and unstable coexistence are viewed as opposite types of coexistence but processes that promote these types of coexistence are difficult to disentangle. Ecological drift is an extreme type of unstable coexistence: equalising processes are no longer needed, and species exclusions are compensated by speciations.

Fig. 1A), because stabilising processes create sites where the better competitor cannot recruit new individuals. Three coexistence mechanisms are based on such processes. In the three cases we consider that there are two species, one of which is a better competitor and would exclude the other without the creation and maintenance of a spatial structure. We show that the three mechanisms correspond truly to coexistence through partition of space itself.

A first mechanism, the earliest mentioned, is the Janzen-Connell hypothesis (Janzen 1970, Connell 1971): seeds and seedlings which are close to their mother-tree are likely to suffer a higher mortality than the ones which are further away. This would be due to the existence of species-specific predators acting more strongly near parent trees or when seed and seedling densities are high, or to the existence of intraspecific competition between seedlings and their mother-tree. This process would be very important to explain tree biodiversity in tropical forest. Although its true significance is still controversial (Clark and Clark 1984, Schupp 1992, Ris Lambers et al. 2002), we emphasise that the invoked mechanism is based on 'holes' made in the surface occupied by the dominant species.

A second mechanism, is based on the existence of a trade-off between colonisation and competitive ability (Levin and Peine 1974, Tilman 1994). Such a trade-off is predicted because of the expected numerous trade-offs influencing allocation of resources to reproduction (Tilman 1990): (1) plants have to produce either a low number of big seeds which should give birth to highly competitive seedlings or a high number of smaller seeds, (2) structures promoting long-distance seed dispersal are resource costly, (3) resources invested in reproduction cannot be invested into the growth or maintenance of the vegetative system. Our point here is that the more space pre-emptive species should have the lower colonising ability, so that it should not be able to disperse seeds to all possible local patches, and is hence locally recruitment limited. This leaves 'holes' where the species with the poorer competitive ability can recruit new individuals whereas, in all patches where some seeds of the better competing species have been dispersed, an individual of this species will be recruited. 
A third mechanism, based on some endogenous heterogeneity and the use of space as a resource, has been recently proved to promote coexistence and has been called heteromyopia (Murell and Law 2003). If the average distance at which conspecific individuals compete with each other is longer than the distance at which heterospecific individuals compete, again 'holes' should be created around individuals of the more competitive species, available for colonisation by the less competitive species. This process should be more intense when the density of the better competitor increases and should always enable the recolonisation of the community by the poorer competitor starting at a low density. This confers stability to coexistence.

The three mechanisms described above are based on the creation of sites in which the poorly competitive species can recruit new individuals. Similarly to coexistence through exogenous heterogeneity, coexistence through endogenous heterogeneity is based on a partition of space, the only difference is that patches available to the different species are not predetermined, but dynamically allocated. It should be possible, in the context of a coexistence model or a real plant community, to measure at each time step the surface corresponding to the sites where each species is more likely to recruit new individuals. These sites would be defined taking into account distances to the neighbours of the different species. It must be noticed that the word 'recruitment' is used here in a broad way: it concerns the transition between two stages which mostly limits the demography of a species (the growth rate of the population), it might be the recruitment of seedlings as well as the recruitment of adults.

\section{Is it possible to disentangle stable and unstable coexistence?}

We have so far described processes that permit plant species to coexist in a stable way: i. e. species densities might show oscillations, but no long term trend is possible, and no species can go extinct: a species driven to a very low density is always able to increase its density again. This has to be checked using the invasibility criterion (Metz et al. 1992). These processes have been coined stabilising processes (Chesson 2000b). Other coexistence processes only allow for unstable coexistence (Fig. 1B). These processes have been called equalising because they decrease fitness differences between individuals of different species, which slows down the exclusion dynamics of poorer competitors (Chesson 2000b) but eventually does not impede exclusion. In that sense, if the species richness of a community is mostly due to equalising factors, the species present at a given time should depend mostly on chance and history (Chesson and Case 1986, Hurtt and Pacala 1995). We show below that it is in fact difficult to disentangle stochastic and deterministic factors involved in coexistence mechanisms and that there is a continuum from perfectly stable coexistence to unstable coexistence.

Coexistence through exogenous temporal variability or spatial heterogeneity is due to the fact that each species is the best competitor in given local environmental conditions which are available during given periods. The stability of coexistence is thus sensitive to the time series of the relevant environmental variables and to the way environmental heterogeneity is structured, i.e. to the spatio-temporal dynamics of favourable sites. If coexistence is very sensitive to these factors, coexistence might be unstable although stabilising processes are active. For example, coexistence might depend on time-partitioning and on a particular alternation of humid and dry years, a stabilising process, but coexistence might stop if a year is unusually dry. Conversely, disturbance-mediated coexistence is traditionally said to belong to unstable coexistence and non-equilibrium theories (Huston 1979, Silvertown and Law 1987). However, this type of coexistence can be viewed to be based on stabilising factors (Fig. 1A). To slow down the exclusion dynamics of the poorer competitors, disturbances have to provide locally and momentarily suitable conditions that allow the poorer competitor to recruit new individuals. This kind of coexistence can thus be related to coexistence through exogenous variability or heterogeneity since disturbances create heterogeneity in both space and time.

Trade-offs and other factors which decrease fitness differences between species should only slow the rate of extinction in poorly competitive species (Chesson $2000 \mathrm{~b}$ ). However, when the fitnesses of two species approaches each other, then the weaker species needs to be stabilizing processes to insure their stable coexistence (Chesson 2000a,b, Murell and Law 2003). One might argue that the colonisation-competition trade-off is classified as a stabilising process. In fact, this trade-off is first an equalising mechanism but since it influences the spatial structure of the population, it generates heterogeneity (local patches where the dominant/dominated species is very likely to recruit new individuals) and is thus also a stabilising mechanism.

Intraspecific spatial aggregation (Fig. 1B) and interspecific spatial segregation, which are very common in plant communities, have first been thought as factors promoting stable coexistence with the rationale that spatial segregation decreases the intensity of interspecific competition and increases intraspecific competition (Pacala and Levin 1997, Stoll and Prati 2001, Murell et al. 2002). However, it is likely that spatial segregation and the formation of conspecific clusters only slows down the exclusion of poorer competitors. It decreases the contacts between heterospecific individuals and thus diminishes the chances of the better competitor to invade 


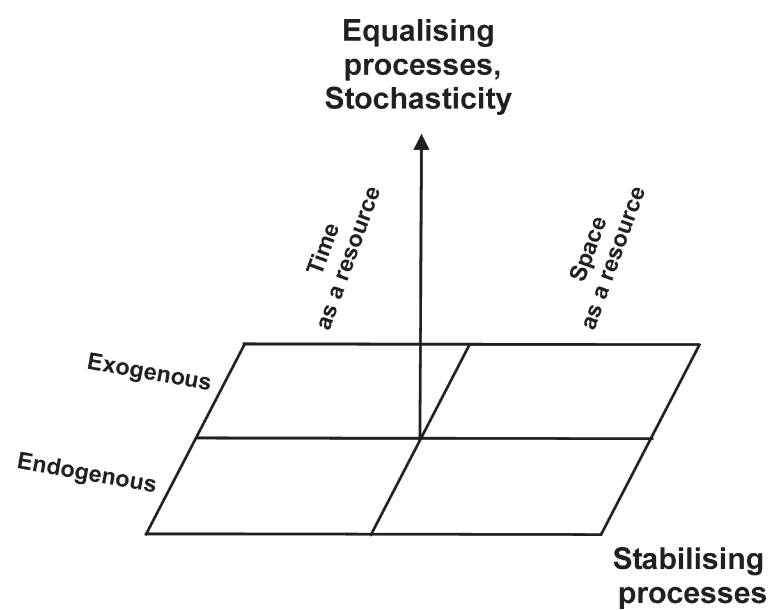

Fig. 2. Synthetic classification of mechanisms of plant coexistence. To describe coexistence in a plant community it must be first determined which resources are involved in stabilising processes (space or time) and whether heterogeneity and variability is self-created by the plant community itself (endogenous) or generated by external factors (exogenous). It must then be assessed, for each resource involved, how much stabilising factors which lead to stable coexistence are intermingled with equalising processes which lead to unstable coexistence.

local patches occupied by the poorer competitor, yet, at the cluster boundaries the species with the better competitive ability should be able to invade the clusters of the poorer competitors (Chesson and Neuhauser 2002). Thus, clusters of the dominant species should slowly exclude clusters of the less competitive species. Taken together, interspecific segregation does not imply stable coexistence but some stabilising processes such as heteromyopia (Fig. 1A) lead to spatial segregation (Murell and Law 2003).

One of the most popular theories to explain the coexistence of numerous tree species in tropical forests is based on recruitment limitation (Hurtt and Pacala 1995). Due to relatively short dispersal distances (whereas dispersal was always global in former models) and to imperfect dispersal (the seed rain is heterogeneous within the dispersal distance of a parent tree) the species with the best fitness of a community cannot send seeds to all available local patches so that species with low competitive ability should win sites by forfeit. As the species richness of a community increases, the proportion of winning-by-forfeit increases. We call these processes 'recruitment limitation as a rule' because no specific stabilising process is involved (doted-line box, Fig. 1B). Field data show that species are indeed often recruitment limited (Condit et al. 1992). On the one hand, using the same rationale as for spatial segregation, it is obvious that recruitment limitation can slow down exclusion of poorer competitors but do not allow for stable coexistence. The species with the most effective combination of vital rates will gain new sites and exclude poorer competitors even though slowly. On the other hand, short dispersal distances and imperfect dispersal (Fig. 1B) are linked to stabilising processes (JanzenConnell hypothesis, colonization-competition trade-off) and more generally to processes (local interactions, local dispersal) that result in complex intra- and interspecific spatial patterns.

Even if stabilising processes create endogenous heterogeneity, when the number of species increase in a community and when population size decreases, stochasticity might override the system. Classical demographic stochasticity might result in the persistence/extinction of a species whatever its vital rates. Here, spatially-mediated demographic stochasticity should add some more stochasticity because there is a priori no guaranty that suitable spatial patterns for coexistence will develop. The persistence of a given species in the community might depend on the formation of a particular spatial structure containing a certain type of local patches (defined by the local distribution of neighbours of the different species) with a suitable frequency. This spatial structure might only appear with a low probability due to underlying stochastic processes. We have thus to conclude that real plant communities display a continuum of possibilities between two theoretical points of view: coexistence through local recruitment limitation that allows for the coexistence of some species through particular deterministic mechanisms, and recruitment limitation as a rule which affects all species and allows for their unstable coexistence (the two doted line boxes of Fig. 1).

Hubbell's theory of ecological drift is even more extreme (Hubbell 2001, Fig. 1C). According to him, there is no dominant species, all species have equivalent fitness because of equalising factors and diversity is determined by the rates of random extinction and speciation events. Hence, coexistence is just an appearance due to ecologists looking at a slow exclusion process within a too short time window relative to process speed; the difference with the previous section is that no equalising mechanism is invoked to slow down the exclusion: the exclusion is slow enough per se. Taken together, it is difficult to disentangle deterministic mechanisms of coexistence and stochastic processes that only slow down exclusion, and there is a continuum of possibilities of coexistence from purely deterministic to perfectly stochastic points of view. This justifies our simplified classification of mechanisms of coexistence (Fig. 2).

\section{What remains to be done}

The goal of this paper is to find a consistency among the numerous coexistence mechanisms now known to be active in plant communities. If a consistent theory of coexistence is to be built, effort should be put on this 
synthesis rather than on lengthening the list of coexistence mechanisms. We propose here a classification that, we hope, could help in such a task. Taking into account all potential mechanisms of coexistence, and all their possible combinations, our first conclusion is that the coexistence of many plant species on the same few resources is no longer a mystery. The classification of mechanisms of coexistence can be simplified as shown by Fig. 2. The classification is based on three axes, two for stabilising processes and one for equalising processes. The two first ones indicate which kinds of resource partitioning that push towards stable coexistence. The third one indicates how stable coexistence is. It must be noticed that several coexistence mechanisms are probably involved in each community, so that the importance of each resource has to be determined (endogenous/ exogenous variability, endogenous/exogenous heterogeneity) and that for each resource the importance of stochasticity has to be assessed.

Our second conclusion is that endogenous variability and heterogeneity offer numerous possible ways promoting coexistence through self-organisation. The multiplicity of these possibilities of coexistence is due to the existence of a feedback loop (above). The whole process of self-organisation leads both to the creations of niches and to the differentiation of niches between species. We can thus answer 'yes' to the question 'Do plants need a niche?' (Chesson 1991), if we accept that plant niches depend on the spatial dynamics of whole plant communities and not only on pre-existing features of physical environment. In this context, although the apparent contradiction between plant biodiversity and the principle of competitive exclusion can be considered as elucidated, and although our understanding of plant communities has highly increased during the last decades, at least four issues are still pretty much open and warrant that researches on plant coexistence should still be fruitful.

\section{Why bother with mechanisms of coexistence?}

Apart from a theoretical point of view, determining which mechanisms that explain coexistence of many plant species in real plant communities is likely to have practical implications. First, the mechanisms through which plants coexist determine in which way species of given communities are complementary, which in turn influences the functional role of biodiversity in these communities (Mouquet et al. 2002). For example, if many species of communities coexist through time partitioning, the insurance hypothesis (Yachi and Loreau 1999) is likely to apply: the diversity of plant reaction to environmental temporal variability diminishes the variability and increases the mean of ecosystem outputs such as primary production. Besides, modifying processes, such as plant spatial pattern or seed dispersal distance, that are involved in coexistence through endogenous heterogeneity modifies the structure of plant communities (relative abundance of species) and consequently alter ecosystem functions (Pacala and Deutschman 1995, Pacala et al. 1996).

Second, it is important to determine how stable coexistence is and whether plant communities (or which communities) are driven by stabilising or equalising processes. It can be predicted that the type of coexistence mechanism that dominates a community determines whether disturbances, such as the loss of a species due to human activities or climatic changes, will have a general negative impact on biodiversity (loss of many species), or a relatively benign impact. This hypothesis should be tested and could have important implications in plant conservation biology and ecosystem management. Of course, achieving this research program and applying its results to real cases is a long-term task since it requires the determination of the respective influence of coexistence mechanisms in real communities (below).

\section{What about real communities?}

Our discussion is mostly based on the results of theoretical models and enables to classify conceptually the different processes that shape the biodiversity of plant communities. It is also important to determine which processes allow for coexistence in real communities. Many studies test for the existence of a particular mechanism of coexistence in a given communities: the Janzen-Connell hypothesis (Condit et al. 1992), recruitment limitation (Hubbell et al. 1999), spatial segregation (Stoll and Prati 2001). However, very few studies test thoroughly for all possible mechanisms, for their relative importance and the way they interact, which would be fundamental to stick to a pluralistic (and probably more realistic) view of ecology. The issue is then to assess the respective influence of each mechanism for different communities and at last to derive a classification of plant communities according to the main processes that shape their biodiversity. Achieving such a research program would also explain why the number of coexisting plant species is varying so much between plant communities and especially between tropical and temperate forests.

Clearly, designing and achieving experiments to test all possible mechanisms of coexistence for even a single community is a huge amount of work. If coexistence is achieved through equalising processes that only slow down the extinction dynamics, human life is probably too short to achieve any relevant experiment. Coexistence through stabilising or equalising processes or through ecological drift might look exactly the same at a 5- or 10-year scale. A solution might be to build a simulation model that encompasses all possible 
mechanisms of coexistence. If such a model is parameterised cautiously using field data and experimental results, the sensitivity analysis should then enable to assess the respective influence of each process and to conduct long-term computer-based experiments which are usually not feasible due to the lengths of human life and research projects (Pacala 1996).

\section{Stochastic and deterministic processes involved in coexistence}

The formation of spatial patterns is intrinsically stochastic, and the likelihood of the appearance of a given complex spatial pattern linked to coexistence is poorly known (Murell et al. 2002), especially when many species are present. It is for example not known how sensitive plant spatial distributions are to the initial conditions of the community settlement, or whether spatial distributions are likely to reach an equilibrium or go through oscillations. This is a key-point to understand coexistence through endogenous heterogeneity based either on stabilising (Fig. 1A) or equalising (Fig. 1B) processes. If realised spatial patterns depend strongly on chance and history, coexistence through endogenous heterogeneity and the nature of co-occurring species itself would depend more on equalising factors and stochastic processes.

In this context, the following hypothesis could be tested: community with low diversity are dominated by deterministic processes while highly diverse communities are dominated by stochastic processes. This should be the case because the dynamics of plant communities with high species richness should be strongly influenced by demographic stochasticity and spatially-mediated demographic stochasticity (Fig. 1B). Practically, methods should also be proposed to assess the 'intensity' or the stability of coexistence in real and modelled communities. This would be achieved by measuring the likelihood of species extinction at various time scales.

\section{Coexistence and evolution}

Although Hubbell's theory is disputable (Ricklefs 2003) it has the advantage to take speciations into account, and more generally the fact that species of plant communities have evolved and are still evolving and that the structure of these communities is both shaped by the historic assembling of species (extinction, immigration), the evolution of already co-occurring species and speciation. Hubbell's theory is relevant only if dynamical processes are slow (slow competitive exclusion of species) and if evolutionary processes are rapid (Ricklefs 2003). However, considering evolutionary processes would probably be useful to understand better species coexistence and predict which mechanisms of coexistence are likely to be the most important. Evolution and competition are tightly intermingled: species that form biodiversity are first created by evolution through speciation and conversely the structure of a plant community (and thus biodiversity) influences competition and thus evolutionary pressures.

First, models of sympatric speciation would probably help to predict which mechanisms of coexistence are likely to evolve. In this case, stabilising coexistence mechanisms should be built in. Second, if we consider that species co-occurring in a community have evolved in an allopatric way, it is important to study the evolutionary consequences of interspecific competition which should promote the evolution of equalising and stabilising processes. On the whole, determining the coexistence mechanisms resulting from different types of speciation and evolutionary pressures remain a pretty open question. New modelling tools, which have been precisely designed to bridge evolutionary and demographic processes, should help to achieve these goals (Dieckmann 1997, Dieckmann and Doebeli 1999).

\section{References}

Abrams, P. A. 1988. How should resources be counted? - Theor. Popul. Biol. 33: 226-242.

Bauer, S., Berger, U., Hildenbrandt, H. et al. 2002. Cyclic dynamics in simulated plant populations. - Proc. R. Soc. Lond. B 269: 2443-2450.

Bolker, B. and Pacala, S. W. 1997. Using moment equations to understand stochastically driven spatial pattern formation in ecological systems. - Theor. Popul. Biol. 52: 179-197.

Chesson, P. 1991. A need for niches? - Trends Ecol. Evol. 6: $26-28$.

Chesson, P. 2000a. General theory of competitive coexistence in spatially-varying environments. - Theor. Popul. Biol. 58: 211-237.

Chesson, P. 2000b. Mechanisms of maintenance of species diversity. - Annu. Rev. Ecol. Syst. 31: 343-366

Chesson, P. L. and Warner, R. R. 1981. Environmental variability promotes coexistence in lottery competitive systems. - Am. Nat. 17: 923-943.

Chesson, P. L. and Case, T. J. 1986. Overview: non-equilibrium community theories: chance, variability, history, and coexistence. - In: Diamond, J. and Case, T. J. (eds), Community ecology. Harper \& Row, pp. 229-239.

Chesson, P. and Neuhauser, C. 2002. Intraspecific aggregation and species coexistence. - Trends Ecol. Evol. 17: 210-211.

Clark, D. A. and Clark, D. B. 1984. Spacing dynamics of a tropical rain forest tree: evaluation of the Janzen-Connell model. - Am. Nat. 124: 769-788.

Condit, R., Hubbell, S. P. and Foster, R. B. 1992. Recruitment near conspecific adults and the maintenance of tree and shrub diversity in a neotropical forest. - Am. Nat. 140: $261-286$.

Connell, J. H. 1971. On the role of natural enemies in preventing competitive exclusion in some marine animals and in rain forest trees. - In: Den Boer, P. J. and Gradwell, G. (eds), Dynamics of populations. PUDOC, Wageningen, pp. 298312.

Crawley, M. J. 1996. Plant-herbivore dynamics.. - In: Plant ecology. - Blackwell Science, pp. 401-474. 
Dieckmann, U. 1997. Can adaptive dynamics invade? - Trends Ecol. Evol. 12: 128-131.

Dieckmann, U. and Doebeli, M. 1999. On the origin of species by sympatric speciation. - Nature 400: 354-357.

Grubb, P. J. 1977. The maintenance of species-richness in plant communities: the importance of the regeneration niche. - Biol. Rev. 52: 107-145.

Hubbell, S. 2001. The unified neutral theory of biodiversity and biogeography. - Princeton Univ. Press.

Hubbell, S. P., Foster, R. B., O’Brien, S. T. et al. 1999. Light-gap disturbances, recruitment limitation, and tree diversity in a neotropical forest. - Science 283: 554-557.

Hurtt, G. C. and Pacala, S. W. 1995. The consequences of recruitment limitation: reconciling chance, history and competittive differences between plants. - J. Theor. Biol. 176: $1-12$.

Huston, M. 1979. A general hypothesis of species diversity. - Am. Nat. 113: 81-101.

Huston, M. A. and DeAngelis, D. L. 1994. Competition and coexistence: the effects of resource transport and supply rates. - Am. Nat. 144: 954-977.

Hutchinson, G. E. 1959. Il concertto moderno di nicchia ecologica. - Mem. Inst. Ital. Idrobiol. 11: 9-22.

Hutchinson, G. E. 1961. The paradox of the plankton. - Am. Nat. 95: 137-145.

Janzen, D. H. 1970. Herbivores and the number of tree species in tropical forests. - Am. Nat. 104: 501-528.

Jones, C. G., Lawton, J. H. and Shachak, M. 1997. Positive and negative effects of organisms as physical ecosystem engineers. - Ecology 78: 1946-1957.

Law, R. and Dieckmann, U. 2000. A dynamical system for neighborhoods in plant communities. - Ecology 81: $2137-$ 2148

Levin, S. A. and Peine, R. T. 1974. Disturbance, patch formation, and community structure. - Proc. Natl. Acad. Sci. 71: 2744-2747.

Metz, J. A. J., Nisbet, R. M. and Geritz, S. A. H. 1992. How should we define'fitness' for general ecological scenarios? - Trends Ecol. Evol. 7: 198-202.

Mouquet, N. and Loreau, M. 2002. Coexistence in metacommunities: the regional similarity hypothesis. - Am. Nat. 159: 420-426.

Mouquet, N., Moore, J. L. and Loreau, M. 2002. Plant species richness and community productivity: why the mechanism that promotes coexistence matters. - Ecol. Lett. 5: 56-65.
Murell, D. J. and Law, R. 2003. Heteromyopia. - Ecol. Lett. 6: $48-59$.

Murell, D. J., Purves, D. W. and Law, R. 2002. Intraspecific aggregation and species coexistence. - Trends Ecol. Evol. 17: 211.

Pacala, S. W. 1996. Dynamics of plant communities.. - In: Plant ecology. - Blackwell Science, pp. 532-555.

Pacala, S. W. and Deutschman, D. H. 1995. Details that matter: the spatial distribution of individual trees maintains forest ecosystem function. - Oikos 74: 357-365.

Pacala, S. W. and Levin, S. A. 1997. Biologically generated spatial pattern and the coexistence of competing species. - In: Tilman, D. and Kareiva, P. (eds), Spatial ecology. Princeton Univ. Press, pp. 185-203.

Pacala, S. W., Canham, C. D., Saponora, J. et al. 1996. Forest models defined by field measurements: estimation, error analysis and dynamics. - Ecol. Monogr. 66: 1-43.

Ricklefs, R. E. 2003. A comment on Hubbell's zero-sum ecological drift model. - Oikos 100: 185-192.

Ris Lambers, J. H., Clark, J. S. and Beckage, B. 2002. Densitydepedent mortality and the latitudinal gradient in species diversity. - Nature 417: 732-735.

Schupp, E. W. 1992. The Janzen-Connell model for tropical tree diversity: population implications and the importance of spatial scale. - Am. Nat. 140: 526-530.

Silvertown, J. and Law, R. 1987. Do plants need niches? Some recent developments in plant community ecology. - Trends Ecol. Evol 2: 24-26.

Stoll, P. and Prati, D. 2001. Intraspecific aggregation alters competitive interactions in experimentals plant communities. - Ecology 82: 319-327.

Tilman, D. 1982. Resource competition and community structure. - Princeton Univ. Press.

Tilman, D. 1990. Constraints and tradeoffs: toward a predictive theory of competition and succession. - Oikos 58: 3-15.

Tilman, D. 1994. Competition and biodiversity in spatially structured habitats. - Ecology 75: 2-16.

Tilman, D. and Pacala, S. 1993. The maintenance of species richness in plant communities. - In: Ricklefs, R. E. and Scluter, D. (eds), Species diversity in ecological communities. Univ. of Chicago Press.

Yachi, S. and Loreau, M. 1999. Biodiversity and ecosystem productivity in a fluctuating environment: the insurance hypothesis. - Proc. Natl. Acad. Sci. 96: 1463-1468. 